\title{
A Focal Mass-Forming Autoimmune Pancreatitis Mimicking Pancreatic Cancer with Obstruction of the Main Pancreatic Duct
}

\author{
Ippei Matsumoto • Makoto Shinzeki • Hirochika Toyama • Sadaki Asari • \\ Tadahiro Goto • Isamu Yamada • Tetsuo Ajiki • Takumi Fukumoto • Yonson Ku
}

Received: 22 March 2011 / Accepted: 5 April 2011 / Published online: 17 May 2011

(C) 2011 The Author(s). This article is published with open access at Springerlink.com

\begin{abstract}
Introduction and background Autoimmune pancreatitis (AIP) is a rare disease that closely mimics pancreatic cancer (PC) in its presentation. It is very important for clinicians to distinguish one from the other because their treatment and prognosis are vastly different. Typical radiological imaging findings, in particular observation of diffusely or segmentally narrowed main pancreatic duct (MPD) with an irregular wall by endoscopic retrograde cholangiopancreatography (ERCP), are essential for making the diagnosis of AIP. On the other hand, MPD obstruction is one of the most frequent features on ERCP.

Case report We report a rare case of a patient with focal mass-forming AIP strongly suspected of being PC because of MPD obstruction on ERCP.

Conclusion It was difficult to distinguish PC from AIP with current diagnostic modalities. We will continue to make an effort to distinguish between the two disorders to prevent unnecessary surgery.
\end{abstract}

Keywords Autoimmune pancreatitis · Pancreatic cancer . Endoscopic retrograde cholangiopancreatography $\cdot$ Main pancreatic duct · Diagnosis

Autoimmune pancreatitis (AIP) is a rare disease that closely mimics pancreatic cancer $(\mathrm{PC})$ in its presentation. It is very important for clinicians to distinguish one from the other because their treatment and prognosis are vastly different. In 2006, the Japanese Pancreas Society proposed clinical diagnostic criteria for AIP based on a combination of clinical, laboratory, imaging, and histological findings. ${ }^{1}$ According to these criteria, typical radiological imaging findings, in particular observation of diffusely or segmentally narrowed main pancreatic duct (MPD) with an irregular wall by endoscopic retrograde cholangiopancreatography (ERCP), are essential for making the diagnosis of

I. Matsumoto $(\square) \cdot$ M. Shinzeki $\cdot$ H. Toyama $\cdot$ S. Asari $\cdot$

T. Goto $\cdot$ I. Yamada $\cdot$ T. Ajiki $\cdot$ T. Fukumoto $\cdot$ Y. Ku

Division of Hepato-Biliary-Pancreatic Surgery, Department

of Surgery, Kobe University Graduate School of Medicine,

7-5-2 Kusunoki-Cho, Chuo-Ku,

Kobe 650-0017, Japan

e-mail: imatsu@med.kobe-u.ac.jp
AIP. On the other hand, MPD obstruction is one of the most frequent features on ERCP.

Here, we report a rare case of a patient with focal massforming (FMF) AIP strongly suspected of being PC because of MPD obstruction on ERCP.

\section{Case Report}

A previously healthy 79-year-old man with epigastric pain was admitted to another hospital. After examination, he was diagnosed as having acute pancreatitis due to a tumor of the pancreatic tail. After treatment for pancreatitis, he was referred to our hospital for further examination and treatment of the tumor. The patient's blood chemistry data were within normal limits except for slightly elevated serum pancreatic amylase (264 IU/l) and lipase levels (430 IU/1). Serum levels of CA19-9 and CEA were both normal. Serum gamma globulin and total IgG were normal, but IgG4 was elevated $(256 \mathrm{mg} / \mathrm{dl})$. Serum autoantibodies and rheumatoid factor were negative. Dynamic CT imaging revealed an irregular mass measuring $40 \times 23 \mathrm{~mm}$ in the tail of the pancreas. The tumor was not enhanced on the arterial phase and slightly enhanced on the portal phase (Fig. 1a). 

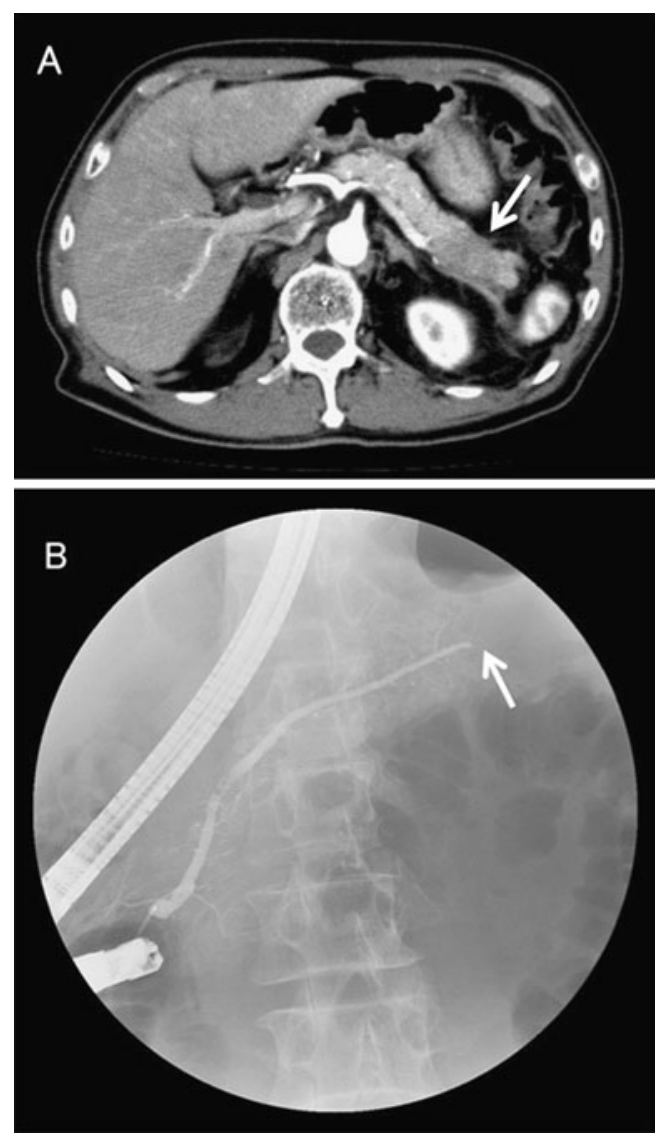

Fig. 1 Dynamic abdominal CT scans in arterial phase showed a lowdensity mass (arrow) measuring $40 \times 23 \mathrm{~mm}$ in the tail of the pancreas (a). ERCP showed an obstruction of the MPD (arrow) at the site of the pancreatic mass (b)

The splenic vein was obstructed by the tumor. MRI imaging showed that the intensity decreased in the T1-weighted images of the pancreas and increased in the T2-weighted images. Endoscopic ultrasonography (EUS) revealed a hypoechoic lesion detected in the tail of the pancreas. EUSguided fine needle aspiration (EUS-FNA), however, did not reveal any cancer cells. 18-Fluorodeoxyglucose positron emission tomography (FDG-PET) showed hot spots of FDG uptake at the site of the pancreatic mass. No extrapancreatic lesions were detected. ERCP revealed an obstruction of the MPD at the site of the tumor (Fig. 1b). Insertion of a guidewire to the distal MPD was impossible. Brush cytology was negative for cancer cells. We suspected PC concomitant with AIP or rather than AIP. The patient underwent a distal pancreatectomy with an uneventful postoperative course.

Gross inspection of the resected specimen revealed a diffusely enlarged and firm pancreas. Histologically, it was remarkable for an intense mixed inflammatory cell infiltrate predominantly composed of lymphocytes and plasma cells, and centered on the pancreatic ducts. The inflammation was associated with significant acinar dropout and parenchymal fibrosis. An obliterative venulitis was noted at the leading edges of the inflammatory cell infiltrate. Immunohistochemical labeling with an antibody to IgG4 revealed large numbers of IgG4-expressing plasma cells.

\section{Discussion}

Although diagnosis of AIP has improved thanks to a growing awareness of the condition and proposed diagnostic criteria, ${ }^{1}$ there remains no practical strategy to differentiate PC from AIP. One must distinguish between the two disorders to prevent unnecessary surgery or delayed initiation of corticosteroid therapy. However, about $3-5 \%$ of patients undergoing pancreatic resection for presumed PC in fact has AIP. ${ }^{2}$ Kamisawa et al. $^{3}$ reported that 7 of 37 (18.9\%) AIP patients had surgery because they were misdiagnosed as having PC or bile duct cancer. In particular, it is very difficult to differentiate between FMF AIP and PC. Chang et al. ${ }^{4}$ reported that 8 of 26 (31.8\%) AIP patients were FMF AIP who were frequently surgically treated because differentiating FMF AIP from PC was so difficult. Kamisawa et al. ${ }^{3}$ also reported that 6 of 17 (35.3\%) FMF AIP patients were surgically treated (resection; 3, bypass operation; 3 ) because PC was suspected.

To obtain images of the pancreatic duct, it is necessary to use ERCP, and additionally direct images taken during the operation or of specimens. Kamisawa et al. ${ }^{3}$ reported that the three ERCP features required for AIP diagnosis were (1) a $>3$-cm-long narrowed main pancreatic duct; (2) skip lesion of the MPD; and (3) maximal upstream MPD diameter of $<5 \mathrm{~mm}$. On the other hand, features highly suggestive of PC were a pancreatic low density mass, MPD obstruction, distal pancreatic atrophy, and metastases. There have been four reports of retrospective evaluation of ERCP imaging in AIP patient. ${ }^{3,5-7}$ The frequency of MPD obstruction on ERCP in AIP patients was $0-5.9 \%$, whereas in PC patients, it was $35-60 \%$, but only three patients with MPD obstruction have been reported. Although the measurement of serum IgG4 level is useful for differentiating between the two diseases, $10 \%$ of $\mathrm{PC}$ patients also has elevated IgG4. ${ }^{8}$ Moreover, there are a few reports of AIP patients with concomitant PC. ${ }^{9,10}$ EUS-FNA is frequently used to rule out PC. However, its accuracy for PC is not perfect (about 70-90\%) because some cases of PC are accompanied by chronic inflammation and fibrosis around the mass, so a negative biopsy does not rule out cancer. Diagnosis of AIP by EUS-FNA is difficult because the specimen is too small. Taken together, we cannot exclude the presence of PC in many cases. Further improvement of diagnostic strategies, such as core biopsy techniques, or development of new immunohistological diagnostic criteria from results of cytologic and tissue specimen analyses are needed to avoid unnecessary surgery. 
In conclusion, we report an extremely rare case of FMF AIP mimicking PC with MPD obstruction. It was difficult to distinguish PC from AIP with current diagnostic modalities.

Open Access This article is distributed under the terms of the Creative Commons Attribution Noncommercial License which permits any noncommercial use, distribution, and reproduction in any medium, provided the original author(s) and source are credited.

\section{References}

1. Okazaki K, Kawa S, Kamisawa T et al. Clinical diagnostic criteria of autoimmune pancreatitis: revised proposal. J Gastroenterol 2006; 41: 626-631.

2. Wolfson D, Barkin JS, Chari ST et al. Management of pancreatic masses. Pancreas 2005; 31: 203-217.

3. Kamisawa T, Imai M, Yui Chen P et al. Strategy for differentiating autoimmune pancreatitis from pancreatic cancer. Pancreas 2008; 37: e62-67
4. Chang WI, Kim BJ, Lee JK et al. The clinical and radiological characteristics of focal mass-forming autoimmune pancreatitis: comparison with chronic pancreatitis and pancreatic cancer. Pancreas 2009; 38: 401-408.

5. Horiuchi A, Kawa S, Hamano $\mathrm{H}$ et al. ERCP features in 27 patients with autoimmune pancreatitis. Gastrointest Endosc 2002; 55: 494-499.

6. Wakabayashi T, Kawaura Y, Satomura Y et al. Clinical and imaging features of autoimmune pancreatitis with focal pancreatic swelling or mass formation: comparison with so-called tumorforming pancreatitis and pancreatic carcinoma. Am J Gastroenterol 2003; 98: 2679-2687.

7. Nishino T, Oyama H, Toki F, Shiratori K. Differentiation between autoimmune pancreatitis and pancreatic carcinoma based on endoscopic retrograde cholangiopancreatography findings. J Gastroenterol 2010; 45: 988-996.

8. Ghazale A, Chari ST, Smyrk TC et al. Value of serum IgG4 in the diagnosis of autoimmune pancreatitis and in distinguishing it from pancreatic cancer. The American journal of gastroenterology 2007; 102: 1646-1653.

9. Inoue H, Miyatani H, Sawada Y, Yoshida Y. A case of pancreas cancer with autoimmune pancreatitis. Pancreas 2006; 33: 208-209.

10. Witkiewicz AK, Kennedy EP, Kennyon L et al. Synchronous autoimmune pancreatitis and infiltrating pancreatic ductal adenocarcinoma: case report and review of the literature. Hum Pathol 2008; 39: 1548-1551. 